\title{
The rationale for including immune checkpoint inhibition into multimodal primary treatment concepts of head and neck cancer
}

\author{
Ingeborg Tinhofer ${ }^{1,2^{*}}$, Volker Budach ${ }^{1,2}$, Korinna Jöhrens ${ }^{3}$ and Ulrich Keilholz ${ }^{4}$
}

\begin{abstract}
Background: Treatment of locally advanced squamous cell carcinomas of the head and neck (SCCHN) remains unsatisfactory. Although the addition of concurrent radiochemotherapy (RCT) or the combination of radiotherapy with blockade of the epidermal growth factor receptor (EGFR) have improved outcomes over radiotherapy alone, further optimization is urgently needed. The introduction of immune checkpoint inhibitors is currently revolutionizing cancer treatment. Clinical evidence has recently been provided in melanoma that immune checkpoint blockade may cooperate with radiation. Therefore, we searched in the literature for the evidence of combining immune checkpoint inhibitors with radiotherapy in primary treatment of SCCHN.
\end{abstract}

Discussion: A substantial amount of previous studies has dissected the molecular mechanisms of immune evasion in SCCHN. The biological effects of radio- and chemotherapy in tumor cells and the immune cell microenvironment were characterized in detail, revealing significant interference of both types of treatment with anti-tumor immunity. This extensive review of the literature revealed considerable amount of evidence that addition of immune checkpoint inhibitors might boost the immunomodulatory potential of radiotherapy and $\mathrm{RCT}$ regimens in SCCHN.

Summary: Promising activity of immune checkpoint inhibitors has already been reported for metastatic/recurrent SCCHN. Given the immunogenic effect of radiotherapy and its enhancement by chemotherapy, combination of radiotherapy or $\mathrm{RCT}$ with this new type of immunotherapy might represent a valuable option for improvement of curative treatment modalities in SCCHN.

Keywords: Radiotherapy, Immune modulation, Combination therapy, Checkpoint inhibitor, Adaptive immunity

\section{Background}

Medical need for improvement of definitive treatment in locally advanced SCCHN

Patients with SCCHN completing radiotherapy-based treatment remain at considerable risk for local relapse within the radiation field, regional recurrence in the neck and hematogenous spread of tumor cells with the potential to

\footnotetext{
* Correspondence: ingeborg.tinhofer@charite.de

${ }^{1}$ Department of Radiooncology and Radiotherapy, Translational Radiation Oncology Research Laboratory, Charite University Hospital Berlin, Charitéplatz 1, 10117 Berlin, Germany

${ }^{2} 2$ German Cancer Research Center (DKFZ), Heidelberg, and German Cancer

Consortium (DKTK) partner site Berlin, Berlin, Germany

Full list of author information is available at the end of the article
}

form distant metastases. Primary radiochemotherapy (RCT) applied concurrently with cisplatin still cures less than $40 \%$ of patients [1], and in case of disease recurrence after RCT, the 2-years survival rate is below $20 \%$. Furthermore, the addition of chemotherapy to radiation improves locoregional control at the cost of severe acute and late morbidity [2] but does not reduce the risk of distant metastases $[1,3]$.

During the last decade, there has been increasing interest in combining RCT with molecularly targeted agents. Most targeted approaches for radiosensitization tested so far have been directed against molecular pathways within cancer cells in order to increase the magnitude of DNA damage or to inhibit cellular mechanisms which interfere 
with tumor cell DNA repair, thereby increasing the efficacy of RCT. Based on the overexpression of EGFR in the majority of SCCHN cases and its causative role in radioresistance $[4,5]$ the EGFR signaling pathway was established as the first molecular target for radiosensitization in SCCHN [6, 7]. Consequently, the combination of cetuximab, a blocking antibody to EGFR with radiotherapy was shown to significantly improve outcome of locally advanced SCCHN when compared to radiotherapy alone [8]. However, despite improvement of locoregional control over radiotherapy alone, the cumulative rate of distant metastasis at 1 or 2 years remained unchanged by this combination [8]. Disappointingly, the RTOG study 0522 evaluating further treatment intensification by combining cetuximab with concurrent RCT failed to meet its endpoints to improve progression-free and overall survival [9]. Further trials which evaluated the combination of RCT with drugs directed against EGFR family members, a broader spectrum of receptor tyrosine kinases or the mTOR signaling pathway have not yet been completed or were stopped early due to the lack of significant activity (Table 1) which underlines the urgent need for novel concepts in this treatment setting. In view of the recent promising results of immune checkpoint inhibitors in the treatment of metastatic/recurrent SCCHN, combination of radiotherapy or RCT with this new type of immunotherapy might represent a valuable option. The aim of this review is to collect evidence from the literature which supports the notion that immune checkpoint blockade may cooperate with radiation in SCCHN.

\section{Basic components of host immunity to cancer}

In principle, the defense by the immune system against pathogenic microbes and toxins from the environment is divided into two general types of processes: the innate immunity and the adaptive immunity. Innate immunity recognizes and fights microbial invaders at the site of

Table 1 Clinical trials evaluating the combination of platinum-based RCT with targeted drugs in locally advanced SCCHN

\begin{tabular}{|c|c|c|c|}
\hline Pathway/Target & Drug & Clinical trial & Results/Status \\
\hline \multicolumn{4}{|c|}{ Tumor-specific targets (terminated trials) } \\
\hline \multirow[t]{4}{*}{ EGFR } & Cetuximab & NCT00265941 (definitive, phase III, RTOG0522) & Negative \\
\hline & Panitumumab & NCT00547157 (definitive, phase II, CONCERT-1) & Negative \\
\hline & Cetuximab & NCT00791141 (adjuvant, phase II, ACCRA-HN) & Not yet reported \\
\hline & Erlotinib & NCT00410826 (definitive, phase II) & Failed to significantly increase CRR or PFS \\
\hline RTK (VEGFR2, EGFR, MET) & Vandetanib & NCT00720083 (adjuvant, phase II, RTOG0619) & Terminated early after 34 pts, no analysis \\
\hline mTOR & Everolimus & NCT00858663 (definitive, phase I) & $\begin{array}{l}\text { Terminated early, only assessment of outcome } \\
\text { at } 6 \text { months - no responses seen }\end{array}$ \\
\hline \multicolumn{4}{|c|}{ Tumor-specific targets (ongoing trials) } \\
\hline \multicolumn{4}{|l|}{ DNA repair } \\
\hline PARP & Olaparib & NCT02308072, (phase I, ORCA-2) & Recruiting \\
\hline \multicolumn{4}{|l|}{ Cell cycle } \\
\hline WEE-1 & AZD1775 & NCT02585973 (phase Ib) & Not yet recruiting \\
\hline CHK-1 & LY2606368 & NCT02555644 (phase I) & Not yet recruiting \\
\hline \multicolumn{4}{|l|}{ EGFR family } \\
\hline EGFR/Her2 & Lapatinib & NCT01711658 (phase II, TRYHARD) & Recruiting \\
\hline \multicolumn{4}{|l|}{ AKT/PI3K } \\
\hline PI3K alpha & BYL719 & NCT02537223 (phase I) & Recruiting \\
\hline Phospho-AKT & Nelfinavir & NCT02207439 (phase II) & Recruiting \\
\hline \multicolumn{4}{|c|}{ Environmental targets (ongoing trials) } \\
\hline Hypoxia & Nimorazole & NCT01880359 (phase III) & Recruiting \\
\hline \multicolumn{4}{|c|}{ Immune checkpoints (ongoing trials) } \\
\hline \multirow[t]{3}{*}{ PD-1 } & Pembrolizumab & NCT02586207, (definitive RCT, phase I) & Recruiting \\
\hline & & NCT02641093 (adjuvant RT or RCT, phase II) & Recruiting \\
\hline & & NCT02296684 (adjuvant RCT, phase II) & Recruiting \\
\hline \multirow[t]{2}{*}{ CTLA-4 } & Ipilimumab & NCT01935921ª (definitive, phase I) & Recruiting \\
\hline & & NCT01860430 (definitive, phase Ib) & Recruiting \\
\hline
\end{tabular}


infection. In contrast, adaptive immunity is serving to eliminate host cells infected with viruses by recognizing peptides from intracellular viral proteins loaded onto major histocompatibility complex (MHC) molecules and displayed on the host cell surface. The adaptive immune system is also able to recognize mutated proteins in tumor cells via the same mechanism.

There are multiple mechanisms by which a tumor cell harboring immunogenic mutations can elicit adaptive immune responses, as schematically summarized in Fig. 1. Tumor cells may spontaneously undergo apoptosis or necrosis, or may be driven to do so by radiotherapy and chemotherapy. The resulting apoptotic bodies can be processed by dendritic cells (DCs). The protein repertoire of dying cells is subsequently presented on the surface to $\mathrm{T}$ cells (the afferent arm of adaptive immune activation). $\mathrm{T}$ cells recognizing peptides derived from 'foreign' mutated proteins are activated by their and, after clonal expansion, these $\mathrm{T}$ cells search throughout the body for tumor cells displaying exactly this mutation on their surface. The cells which are recognized as carrying this mutation are killed through the lytic machinery of $\mathrm{T}$ cells (the execution of the efferent arm of adaptive immunity). However, in a patient with a growing tumor, this system has obviously failed as a consequence of one or many mechanisms which tumor cells have adopted to escape immune destruction.

Immune evasion in SCCHN: hideout, defense, camouflage and balanced immune destruction

There are several ways for SCCHN to evade recognition by the adaptive immune system, as schematically

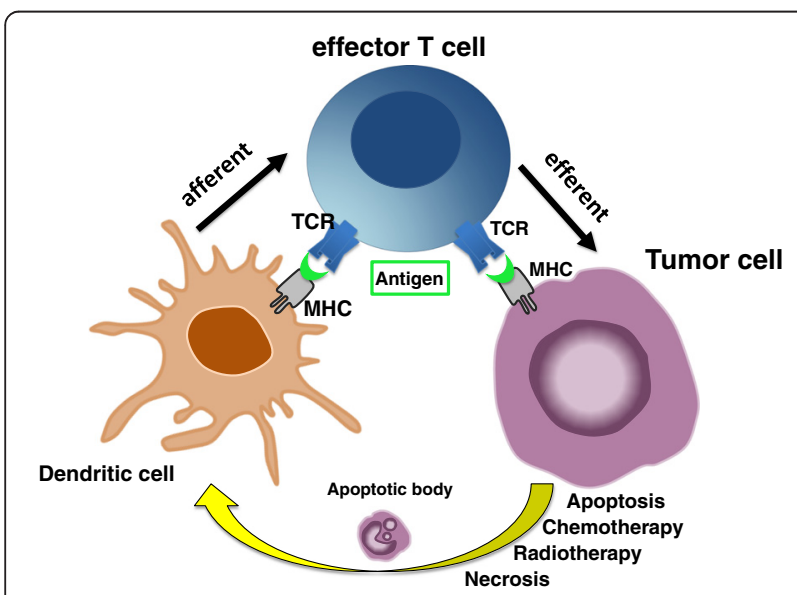

Fig. 1 The afferent and efferent arms of adaptive tumor immunity. Tumor cells undergo apoptosis or necrosis, either spontaneously or after radio- or chemotherapy. Apoptotic bodies from tumor cells can be processed by dendritic cells. The antigen repertoire of dying cells is subsequently presented by dendritic cells via MHC molecules to $T$ cells (the afferent arm of adaptive immune activation). T cells recognizing peptides by their $T$ cell receptor (TCR) are activated and acquire cytolytic effector functions illustrated in Fig. 2. Early tumors may grow in a hideout, because they display neither a significant level of apoptosis or necrosis nor inflammatory signals and thus do not elicit any danger signal in the tissue (Fig. 2a). Although danger signals might subsequently be produced during the progression of the disease, the lymphocytic infiltrate may be confined to the rim of the tumor tissue with no infiltration into the tumor itself (Fig. 2b). Secretion of indoleamine 2, 3 -dioxygenase (IDO) is among the major defense mechanisms used by tumors to prevent lymphocytic infiltration [10]. Tumors frequently also counterbalance infiltration by lymphocytes by down-regulation of their MHC molecules, thereby avoiding the presentation of peptides from intracellular proteins to $\mathrm{T}$ cells which results in an effective camouflage (Fig. 2c). As schematically depicted in Fig. 2d, in tumors with extensive inflammatory cell infiltration a delicate balance between immune destruction and immune evasion may exist which is based on immunosuppressive mechanisms including high expression of IDO and PD-L1 as well as recruitment of FoxP3+ regulatory $\mathrm{T}$ cells (Treg) [11]. Representative histological images from SCCHN tumor sections exemplifying the above-mentioned types of immune evasion are presented in Fig. 3.

A number of both genetic and environmental mechanisms allow such immune escape and have been described in SCCHN (for a recent detailed review see [12]), including selection of poorly antigenic cancer cell subsets, disturbances in MHC class I- and class II-mediated antigen presentation [13-15], expression and secretion of immunosuppressive cytokines [16], expression of the proapoptotic Fas ligand to induce activation-induced cell death in T cells [17], and recruitment of immunosuppressive immune cell subsets into the tumor [18]. More recently, evidence is increasing that expression of immune checkpoint components that may limit $\mathrm{T}$ cell responses also occurs frequently in these tumors [18-20].

\section{Immunomodulatory effects of ionizing radiation}

Ionizing radiation has been used for more than a century to treat cancer [21], on the basis that rapidly proliferating cancer cells are more sensitive to DNA damage induced by radiation than normal tissue. Radiotherapy has traditionally been viewed as immunosuppressive due to the inherent sensitivity of lymphocytes to radiation-induced damage but it became evident that radiotherapy can also enhance tumor-specific immune responses. Strong support of an active role of the immune system for the success of radiotherapy came from studies in which the extent of tumor control by radiotherapy was compared in immunocompetent and -deficient xenograft models. Studies in the model of melanoma revealed that the ablative effects of radiotherapy were strongly dependent on radiation-induced cytokine responses [22] 


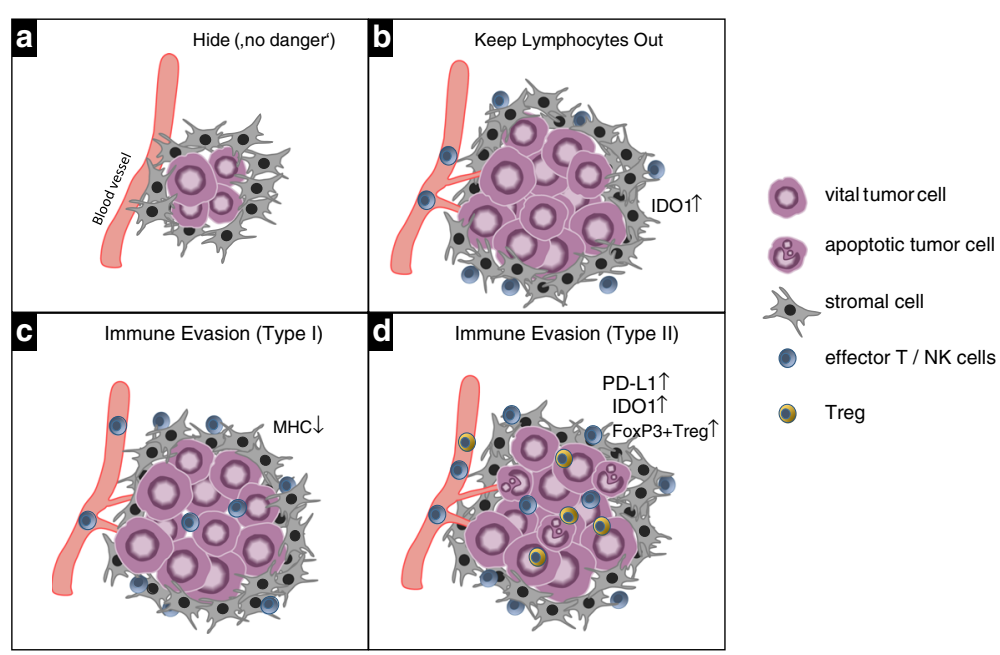

Fig. 2 Mechanisms of immune evasion by tumors. a In the early phase of tumor development tumors remain undetected by the immune system because of the lack of danger signals such as significant levels of apoptotic or necrotic cells or pro-inflammatory cytokines. b By secretion of soluble factors such as indoleamine 2,3-dioxygenase (IDO) by tumor cells the infiltration of lymphocytes is inhibited. c If moderate immune cell infiltration eventually occurs tumor cells downregulate the expression of components of the antigen presentation machinery including MHC class I and II which results in their impaired recognition by antigen-specific $T$ cells. $\mathbf{d}$ In tumors with a larger extent of immune cell infiltration, tumor cell destruction by cytotoxic T cells is inhibited by high expression of immunosuppressive mechanisms such as IDO, PD-L1 and FoxP3+ Treg

and cytotoxic CD8+ T cells [23]. In a preclinical model of SCCHN, pretreatment of tumor cell lines with chemotherapeutic drugs and radiation significantly increased the extent of their cytolysis by antigen-specific CD8+ T cells [24]. All these observations suggest that not only genetic and phenotypic traits of tumor cells but also immunity of the host are implicated into the clinical success of radiotherapy [22, 23, 25-27].

Mechanistically, radiotherapy has been shown to augment the afferent as well as efferent arms of cancer immunity. The induction of a specific $\mathrm{T}$ cell response to tumor cells (afferent immunity) has been observed in

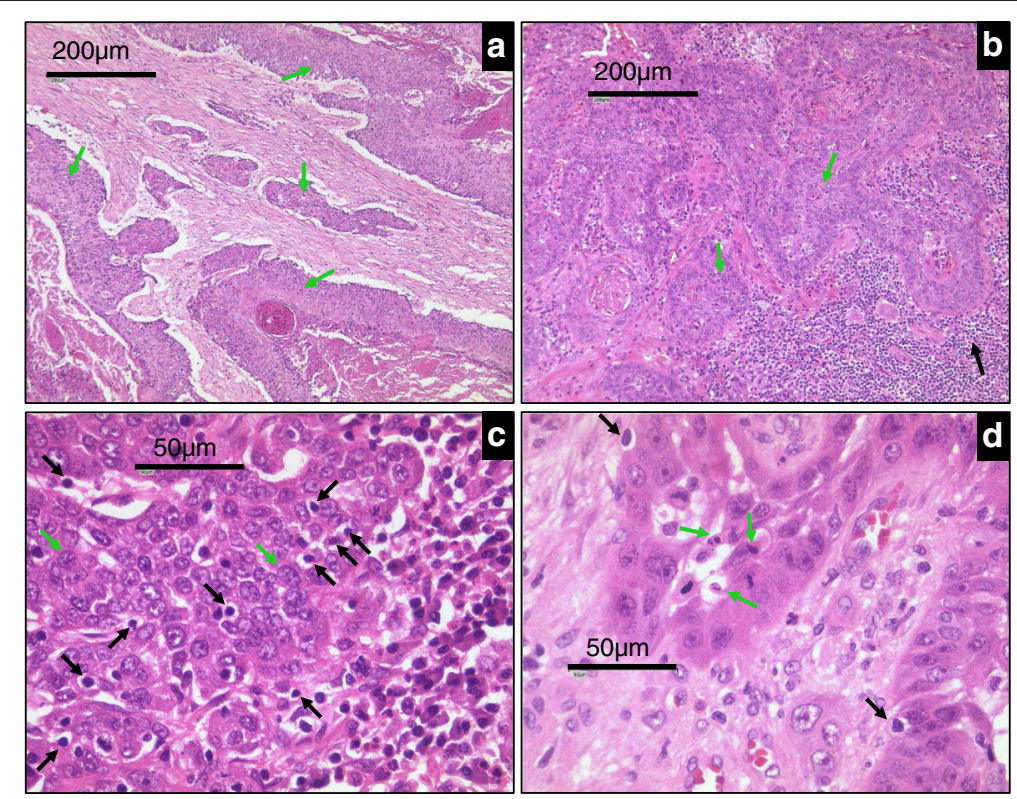

Fig. 3 Representative histological images of SCCHN tumor sections displaying different levels of immune evasion. a Tumor areas (green arrows) show the absence of any lymphocyte filtration at the rim or within the tumor cell nests. $\mathbf{b}$ Lymphocyte infiltrates are seen at the tumor border (black arrows) but are absent within the tumor nests (green arrows). c Despite a high extent of lymphocyte infiltration no signs of tumor cell lysis or apoptosis are visible. $\mathbf{d}$ Tumor areas with infiltrating lymphocytes are composed of vital and apoptotic tumor cells (black arrows), indicative of a balance between immune destruction and evasion 
multiple studies. Almost 20 years ago molecular pathways were first described that were activated by treatmentinduced cell stress (in particular after treatment with anthracyclins and ionizing radiation) and which induced a modality of cell death that was highly efficient in eliciting immune responses [28]. The immunogenic effects of radiation (reviewed in [29]) are thought to result from 'autovaccination' by antigens released from dying tumor cells. Translocation of a protein called calreticulin which is normally residing in the endoplasmatic reticulum to the cell surface promotes the uptake of dying cancer cells by DCs and the release of antigens that can be efficiently presented [30]. Release of ATP, heat shock proteins and high-mobility group box 1 (HMGB1) by dying cancer cells help in recruiting and activating DCs through toll-like receptor signaling pathways [31]. By integration of these danger signals DCs undergo an important maturation process. They upregulate the expression of co-stimulatory proteins and pro-inflammatory cytokines, and acquire the ability of cross-presenting antigens to cytotoxic CD8+ $\mathrm{T}$ cells by which they initiate adaptive immunity [32].

Radiotherapy can also influence the efficacy of tumor cell destruction (efferent immunity) within the radiation field by altering tumor cell characteristics or the tissue microenvironment. Tumor cells in which the damage from radiation has not been sufficient to induce cell death show increased expression of MHC class-I antigenpresenting molecules [33] and adhesion molecules [34], stabilizing the binding of $\mathrm{T}$ cells to tumor cells and alleviating TCR activation. As a result, tumor cells that survive radiation may be eliminated through $\mathrm{CD} 8+\mathrm{T}$ cell-mediated lysis [33].

It has been known for some time that irradiated tissues often show very strong changes in the local cytokine milieu. As a result of their action, a cascade of pro-inflammatory processes are triggered. Secretion of interferon- $\gamma$ enhances expression of MHC class-I by cancer cells, sustaining and extending the initial effects of radiation to allow efficient recognition and killing by $\mathrm{T}$ cells $[26,34]$. In addition, immune cell trafficking is enhanced through induction of chemokines, such as CXCL16 that attract effector T cells to the irradiated tumor site [29].

Immune effects within the radiation field, however, are not sufficient for cure, as effective treatment of a highrisk primary cancer has to secure not only local but also systemic control of the disease. In principle, the nature of the adaptive immune system should be mechanistically well suited for systemic tumor surveillance. There is emerging evidence that radiotherapy can also be associated with immune destruction of distant metastases, pointing towards efferent immunity outside of the radiotherapy field. This phenomenon termed abscopal antitumor effect was already described in 1953 [35]. Clinical reports of an abscopal effect after radiotherapy are few, but cover several different tumor types, including melanoma and a variety of carcinomas [36]. Growth suppression of distant non-irradiated tumors by a combination therapy of DC infusion and radiotherapy was also reported in a murine model of squamous cell carcinoma in the head and neck, indicating that indeed systemic antitumor activity can be induced by approaches which augment the immune-activating effects of radiotherapy.

The knowledge gained from mechanistic studies on the immunomodulatory effects of radiation mentioned above has changed the way the response to radiotherapy with/without chemotherapy in patients with cancer is now interpreted, by acknowledging the essential role of the host immune system for the success of radiotherapy. Importantly, these indirect effects of radiation - within and outside the field of treatment - also suggest new treatment possibilities, including combinations with established or novel forms of immunotherapy.

\section{Immune checkpoint blockade as novel immunotherapeutic strategy in cancer}

The introduction of immune checkpoint inhibitors is currently revolutionizing treatment of metastatic cancers. Previously, cancer immunology had concentrated either on afferent immune stimulation, i.e. induction of $\mathrm{T}$ cell immunity, most frequently by vaccination, or on stimulation of efferent $\mathrm{T}$ cell activity, e.g. by interleukin-2 treatment. An important limitation of these approaches was the tight regulation of the immune system by mechanisms termed immune checkpoints which are physiologically crucial to prevent autoimmune diseases (Fig. 4). At the afferent side of immunity the molecule cytotoxic T-lymphocyte protein 4 (CTLA-4) is expressed on antigen-activated T cells to dampen the magnitude of $\mathrm{T}$ cell activation. At the efferent side, the expression of the cell surface receptor PD-1 (programmed cell death protein 1) on activated T cells block their effector function, if bound to the ligand PD-L1 or PD-L2 on the target cell. Tumor cells frequently use the expression of PD-L1 and PD-L2 to escape immune destruction. Blocking antibodies directed to the immunoregulatory proteins CTLA-4, PD-1 and PD-L1 have been shown to release these immune checkpoints in different ways. Antibodies to CTLA-4 (namely ipilimumab and tremelimumab) allow induction of autoimmunity, including immunity to cancer. However, there is a tight window of opportunity, as autoimmune phenomena can be quite serious after application of these agents [37]. Antibodies to PD-1 or PD-L1 do not promote induction of de-novo immunity but release the effector phase of immunity (Fig. 4), hereby allowing the execution of tumor cell destruction by $\mathrm{T}$ cells. Thus, the presence of tumor-specific $\mathrm{T}$ cells is required for efficacy of agents interfering with the PD-1/PD-L1 interaction. 


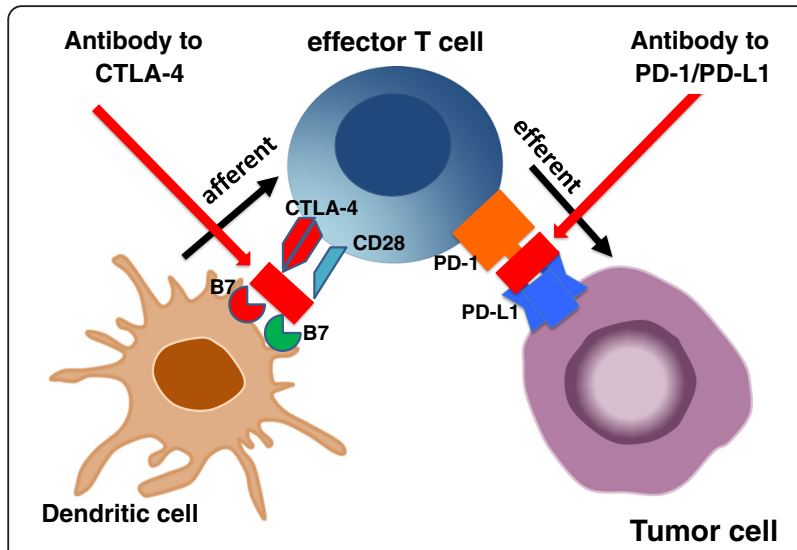

Fig. 4 Immune checkpoints as modulators of the afferent and efferent arm of adaptive immunity. Cytotoxic T-lymphocyte protein 4 (CTLA-4) is an inhibitory receptor acting as a major negative regulator of $T$ cell responses. As part of the afferent immune response CTLA-4 upregulation on antigen-activated $T$ cells dampens the magnitude of $\mathrm{T}$ cell activation. At the efferent side, programmed death receptor 1 (PD-1) which is expressed on activated $T$ cells blocks their effector functions upon binding to the ligands PD-L1 or PD-L2 on target cells. Tumor cells frequently use the expression of PD-L1 and PD-L2 to escape immune destruction

The application of immune checkpoint inhibitors has recently been evaluated in a number of clinical trials and demonstrated remarkable activity in a broad spectrum of cancer types. Ipilimumab, nivolumab and pembrolizumab (the latter two agents both anti-PD-1 antibodies) were the first three immune checkpoint inhibitors which received FDA approval for the treatment of metastatic melanoma. A three-arm phase III trial in melanoma [38] answered the fundamental question in cancer immunology as to whether the de-novo induction of $\mathrm{T}$ cell responses by ipilimumab or the augmentation of a pre-existing $\mathrm{T}$ cell response by nivolumab may be more efficacious. Response rates and progression-free survival clearly favored nivolumab over ipilimumab, with the combination of both even more effective but at the cost of considerable immunerelated toxicities [38].

There are at least eight anti-PD-1/PD-L1 antibodies currently in clinical development, covering phases I to III. In addition, the preclinical and early clinical development of inhibitors against other immune checkpoints, such as $\mathrm{T}$ cell immunoglobulin mucin receptor 3 (TIM3) and lymphocyte activation gene 3 protein (LAG3), and against co-stimulatory molecules, such as OX40 and CD137, are underway. Final results from several successful phase III trials with ipilimumab, nivolumab and pembrolizumab improving overall survival of metastatic cancer have been reported in melanoma and lung cancer, and it can be expected from the data available for a broad range of other histologies that this novel class of agents will be firmly established in modern treatment of many cancers.
In recurrent/metastatic SCCHN, several PD-1/PD-L1 blocking agents are currently being investigated, with most mature information on nivolumab and pembrolizumab. The phase $1 b$ multicohort trial Keynote-012 tested the efficacy of the anti-PD-1 antibody pembrolizumab for treatment of $\mathrm{PD}-\mathrm{L} 1+$ in recurrent/metastatic SCCHN [39]. A best overall response rate of $18 \%$ was reported, with no obvious difference being observed between $\mathrm{HPV}+$ (25\%) and HPV- tumors (14\%). Duration of responses was approximately 12 months [39]. Comparable results (overall response rate: $18 \%$ HPV+, $22 \%$; HPV-, $16 \%$ ) were reported for the Keynote-055 study in patients with R/M SCCHN resistant to platinum and cetuximab have been included [40]. Moreover, the randomized global phase III trial Checkmate-141, evaluating the efficacy and safety of nivolumab versus investigator's choice in patients with R/M SCCHN demonstrated an increase in 1-year overall survival (OS) rate from 16 to $36 \%$ by nivolumab [41, 42]. Again, a survival benefit was observed in the $\mathrm{HPV}+$ and HPV- subgroup [41, 42].

Early evidence of clinical activity in SCCHN were also reported from multi-arm expansion studies of anti-PDL1 antibodies (atezolizumab, MPDL3280A [43]; durvalumab, MEDI4736 [44]). Based on these promising data, several further randomized phase III trials (NCT02358031, Keynote-048; NCT02252042, Keynote-040) have been initiated. In general, the successful clinical trials of PD-1 blocking agents are a proof of the existence of adaptive immunity towards SCCHN cells which can be very effective in a proportion of patients when unleashed by blockade of the PD-1/PD-L1 interaction.

\section{Interference of immune checkpoints with resistance to RCT}

Deregulated expression of immune checkpoint proteins has already been linked to poor efficacy of RCT in several tumor models. High expression of PD-L1 in tumor cells and stromal lymphocytes accompanied by low CD8+ $\mathrm{T}$ cell infiltration has recently been identified as a poor prognostic biomarker in patients with stage III non-small cell lung cancer (NSCLC) receiving cisplatin-based RCT [45]. In addition, tumor control by neoadjuvant or concurrent RCT was observed to be inefficient in patients with esophageal squamous cell carcinomas displaying elevated immunostaining for PD-L1 in neoplastic and adjacent non-malignant esophageal epithelium [46]. Preclinical studies in a variety of syngeneic mouse models of cancer [47] have also demonstrated that expression of PDL1 can be induced by radiation itself and that such upregulation impairs both local tumor control and protection against tumor re-challenge [47]. It is therefore not surprising that blocking antibodies directed to these immune checkpoints were able to significantly enhance the immunogenic effects of radiotherapy [27, 48, 49]. 
In locally advanced SCCHN the magnitude of immune suppression could also be linked to the efficacy of RCT, however, a direct role of immune checkpoint proteins remains to be established. Low numbers of tumor-infiltrating cytotoxic CD8+ T cells before treatment were significantly correlated with poor outcome after RCT in several studies [50-53] but the role of CTLA-4 or PD-1/PD-L1 as negative regulators of $\mathrm{CD} 8+\mathrm{T}$ cell activation has not been addressed. By inducing a change in the composition and functions of the immune cell compartment RCT was shown to relieve the extent of immune suppression: while the numbers of $\mathrm{CD} 8+$ and granzyme $\mathrm{B}+$ cytotoxic cells only slightly decreased after RCT, a more pronounced decrease of FoxP3+ Treg was observed, resulting in an 2to 3 -fold increase in the cytotoxic $\mathrm{T}$ cell/FoxP3+ Treg ratio [50]. These data strongly support the idea that application of immune checkpoint inhibitors together with radio(chemo)therapy could also lead to a significant improvement of local as well as distant tumor control in SCCHN. Consequently, the first phase I/II trials evaluating the combination of pembrolizumab with standard definitive or adjuvant RCT as well as ipilimumab with cetuximabbased bioradiation (Table 2) have already been started, and several further trials with other inhibitors of PD-1 or PD-L1 are in preparation.

\section{Potential biomarkers for patient selection for immune checkpoint blockade}

Precise biomarkers to identify patients who benefit from immune checkpoint inhibition alone or in combination with RCT still have to be established. Current data in multiple cancers reveal that verification of PD-L1 overexpression by immunohistochemistry is associated with improved clinical outcome of anti-PD-1 therapy. However, the presence of robust responses in some patients with low or undetectable expression of PD-L1 complicates the issue of PD-L1 as an exclusive predictive biomarker [54].
In the Keynote-012 trial of SCCHN, an elevated expression of PD-L1 and the presence of an interferon- $\gamma$ expression signature were associated with improved progression-free survival $[39,55]$. The same signature had previously been established as predictive signature for outcome after pembrolizumab in metastatic melanoma [56] and its predictive value was also demonstrated in advanced gastric cancer [57]. In addition, patients with large immune cell infiltration in tumor tissue and high mutational load were more likely to benefit from immune checkpoint blockade in bladder [58] and colorectal cancer [59]. Taken together, patients with PD-L1+ tumors displaying immune-related gene expression signatures, including genes regulating $\mathrm{T}$ cell functions, the antigen presentation machinery and IFN- $\gamma$ signaling, are most likely to benefit from immune checkpoint inhibition.

\section{Rational development of combination regimens}

While there is much excitement around the phenomenon of a radiation-induced anticancer immune response and combining radio(chemo)therapy with immunotherapy, numerous questions remain to be addressed in clinical trials. A major challenge is to identify not only the optimal immune checkpoint inhibitor as partner for a given radiotherapy schedule but also the best chronological sequence for their combined application. Preclinical evidence can serve as guidance in treatment schedule and clinical trial development. As outlined above, danger signals induced by radiation lead to the recruitment of immune cells into the tumor. However, cells of the immune system are also vulnerable to radiation, as their exposure to ionizing radiation induces apoptosis in mature natural killer (NK) cells as well as T and B cells. Since radiotherapy is generally delivered in daily fractions, re-irradiation of the tumor might therefore damage infiltrating immune cells that display cytolytic activity themselves or might significantly reduce the capacity of DCs to activate effector T cells. In addition,

Table 2 Current clinical trials (at clinicaltrials.gov) evaluating the combination of RT with immune checkpoint inhibitors

\begin{tabular}{|c|c|c|c|}
\hline Clinical setting & Clinical trial & Drug & Combination \\
\hline \multirow[t]{2}{*}{ Resectable locally advanced SCCHN } & NCT02641093 phase II & Pembrolizumab & Adjuvant RT/RCT \\
\hline & NCT02296684 phase II & Pembrolizumab & Adjuvant RT/RCT \\
\hline Locally advanced SCCHN & NCT01935921 phase I & Ipilimumab & Definitive RT + cetuximab \\
\hline Intermediate/High risk locally advanced SCCHN & NCT01860430 phase lb & Ipilimumab & Definitive RT + cetuximab \\
\hline Locally advanced SCCHN & NCT02586207 phase I & Pembrolizumab & Definitive RT + CDDP \\
\hline locally advanced laryngeal carcinoma & NCT02759575 phase I/II & Pembrolizumab & Definitive RT + CDDP \\
\hline \multirow[t]{2}{*}{ Intermediate/High risk locally advanced SCCHN } & NCT02764593 phase I & Nivolumab & Definitive RT, RT+ CDDP, RT + cetuximab \\
\hline & Phase III & Nivolumab & Definitive RT + CDDP \\
\hline locoregional inoperable recurrence or second primary SCCHN & NCT02289209 phase II & Pembrolizumab & Reirradiation \\
\hline Advanced metastatic disease (multicohort) & NCT02303990 phase I & Pembrolizumab & RT \\
\hline Brain metastasis (multicohort) & NCT02669914 phase II & Durvalumab & Stereotactic radiosurgery \\
\hline
\end{tabular}

$R T$ radiotherapy, $C D D P$ cisplatin 
DCs may find a hostile environment for $\mathrm{T}$ cell activation in draining lymph nodes, which represent their natural surrounding for interaction with $\mathrm{T}$ cells, as draining lymph nodes are systematically included into the radiation field in SCCHN. Conversely, however, the induction of immunogenic cell death (ICD) by each daily radiotherapy fraction might transiently generate a favorable milieu for immune activation within the tumor tissue, which may vanish again after termination of radiotherapy. In support of the latter, concurrent application of an anti-PD-L1 antibody together with fractionated radiotherapy significantly improved tumor control in a xenograft model [47]. In contrast, fractionated radiotherapy followed by delayed application of anti-PD-L1 was completely ineffective in enhancing the local efficacy of radiotherapy [47]. Certainly, more studies will be needed to address this important issue.

Besides the optimal time schedule also the optimal type and dosing of chemotherapy has to be established, if desired to be included into the treatment regimen. Significant differences in the ability of chemotherapeutic drugs to induce ICD have been reported previously [32]. Cisplatin which is an essential component of current state-of-the-art RCT regimens in SCCHN does not induce ICD [60] despite its presumed identical mechanism of action to that of oxaliplatin, a potent inducer of ICD. This has been attributed to the lack of calreticulin exposure after cisplatin treatment [60]. However, radiotherapy is a potent inducer of calreticulin exposure [30], and recent studies have shown that combining cisplatin with compounds that induce calreticulin exposure leads to fullscale ICD [60]. Thus, potentiation of ICD by cisplatin could still represent one of its major mechanism of action when cisplatin is administered concurrently with radiotherapy.

Taxanes including docetaxel and paclitaxel which are also common combination partners of radiotherapy in locally advanced SCCHN are known to modulate antitumor immune responses as well [61]. Similar to cisplatin, paclitaxel does not induce ICD. However, concurrent paclitaxel treatment was shown to significantly enhance radiation-induced ICD in breast cancer cell lines [62]. Similarly, docetaxel treatment itself did not induce ATP or HMGB1 secretion by tumor cells. However, calreticulin exposure of tumor cells after docetaxel treatment was observed which significantly enhanced tumor cell killing by antigen-specific CD8+ cytotoxic T cells [61].

Intriguingly, investigations on the immunogenic effects of chemotherapeutic drugs revealed also their direct interference with immune checkpoint expression. Treatment of DCs in vitro with platinum-based compounds strongly enhanced their potential to activate $\mathrm{T}$ cells which was caused by downregulation of PD-L2 in DCs [63]. This effect was mediated by inactivation of
STAT6, the transcriptional regulator of PD-L2, and occurred also in tumor cells resulting in their enhanced recognition by $\mathrm{T}$ cells [63].

Overall, these preclinical observations provide a sound rationale for investigating immune checkpoint inhibitors with radiotherapy alone as well as in combination with standard cisplatin-based as well as taxane-based RCT. In addition, there is accumulating data that the efficacy of cetuximab-based regimens in treatment of recurrent/ metastatic SCCHN is not only based on the inhibition of EGFR signaling pathways but also on the activation of $\mathrm{Fcy}$ receptor-positive NK cells leading to DC maturation and activation of cytotoxic T cells [64]. A combination of immune checkpoint inhibitors with cetuximab-based bioradiation protocols might therefore also represent a very attractive chemotherapy-free concept for improvement of primary treatment of locally advanced SCCHN.

\section{Toxicity of combination regimens}

The toxicity of radiotherapy is mostly occurring directly at the irradiation site. Mucositis, xerostomia and swallowing dysfunctions are common side effects in radiotherapy of head and neck cancers. Clearly, the extent of early and late toxicity is dependent on the radiation technique and the applied dose: Intensity-modulated radiotherapy, which conforms closely to the tumor volume, avoids or minimizes exposure to unaffected tissue and thereby significantly reduces local side effects of irradiation [65]. On the other hand, addition of concurrent chemotherapy to radiotherapy not only improved efficacy of the treatment in locally advanced SCCHN but also increased both the toxicity and the spectrum of adverse events as compared to radiotherapy alone [66]. The toxicity of immunotherapy is dependent on the administered agent and dosage. In previous clinical trials, immune checkpoint blockade immunotherapy presented acceptable toxicity. Even occasional severe toxicity was manageable through treatment interruption or involvement of immunosuppressive drugs. During ipilimumab treatment approximately $60 \%$ patients showed immunerelated adverse events, of them $10-15 \%$ being grade 3-4 [67]. The blockade of PD-1/-L1 showed less severe ir-AEs in previous phase I studies [68]. Diarrhea and skin rash were the most common immune-related adverse events after ipilimumab, other adverse effects included enterocolitis, hypothyroidism, hypophysitis and neuropathies [68]. The most common adverse events reported for both nivolumab and pembrolizumab were mild fatigue, rash, pruritus and diarrhea, which could be usually managed without dose interruption or discontinuation [68]. First toxicity data from a phase I study of the combined application of ipilimumab with radiotherapy for treatment of metastatic melanoma (NCT01497808, [48]) argue against an exacerbating toxicity profile of the 
combined regimen. No dose-limiting toxicities, defined by the study protocol as any treatment-related grade $\geq 4$ immune-related toxicity or grade $\geq 3$ non-immune related toxicity experienced during study treatment or within 30 days after the last injection of ipilimumab were observed [48]. Considering the different kinds and acceptable adverse events, the combinatorial treatment of radiotherapy and immune checkpoint inhibitors seems feasible for SCCHN patients.

\section{Conclusions}

The introduction of immune checkpoint inhibitors into cancer treatment has been celebrated as the breakthrough of the year 2013. Impressive activity was proven in metastatic melanoma and lung cancer, and promising results were presented for recurrent/metastatic SCCHN. Given the immunogenic effect of radiotherapy and its enhancement by chemotherapy or cetuximab, it remains to be determined whether immune checkpoint inhibitors could further increase the activation of adaptive immunity and ultimately improve overall current cure rates of locally advanced SCCHN.

\section{Abbreviations \\ CTLA-4, cytotoxic T-lymphocyte protein 4; DCs: dendritic cells; DNA, deoxyribonucleic acid; EGFR, epidermal growth factor receptor; HMGB1, high-mobility group box 1; ICD, immunogenic cell death; IDO, indoleamine 2:3-dioxygenase; LAG3, lymphocyte activation gene 3 protein; MHC, major histocompatibility complex; NK, natural killer; NSCLC, non-small cell lung cancer; PD-1, programmed cell death receptor 1; PD-L1, programmed cell death 1 ligand 1; RCT, radiochemotherapy; SCCHN, squamous cell carcinoma of the head and neck; TCR, T cell receptor; TIM3, T cell immunoglobulin mucin receptor 3 ; Treg, regulatory $T$ cells}

\section{Authors' contributions}

$I T, V B, K J, U K$ conception and design. IT and UK literature search and review. IT, VB, KJ, UK interpretation of data. IT and UK manuscript drafting and writing. IT, VB, KJ, UK revision of manuscript and approval of final version.

\section{Competing interest}

The authors declare that they have no competing interests.

\section{Consent for publication}

Not applicable.

Ethics approval and consent to participate

Not applicable.

\section{Author details}

'Department of Radiooncology and Radiotherapy, Translational Radiation Oncology Research Laboratory, Charite University Hospital Berlin, Charitéplatz 1, 10117 Berlin, Germany. ${ }^{2} 2$ German Cancer Research Center (DKFZ), Heidelberg, and German Cancer Consortium (DKTK) partner site Berlin, Berlin, Germany. ${ }^{3}$ Institute of Pathology, Charite University Hospital, Berlin, Germany.

${ }^{4}$ Comprehensive Cancer Center, Charité University Hospital, Berlin, Germany.

Received: 19 February 2016 Accepted: 1 August 2016

Published online: 10 August 2016

\section{References}

1. Pignon JP, le Maitre A, Maillard E, Bourhis J, Group M-NC. Meta-analysis of chemotherapy in head and neck cancer (MACH-NC): an update on 93 randomised trials and 17,346 patients. Radiother Oncol. 2009;92(1):4-14.
2. Machtay M, Moughan J, Trotti A, Garden AS, Weber RS, Cooper JS, Forastiere A, Ang KK. Factors associated with severe late toxicity after concurrent chemoradiation for locally advanced head and neck cancer: an RTOG analysis. J Clin Oncol. 2008;26(21):3582-9.

3. Budach V, Stuschke M, Budach W, Baumann M, Geismar D, Grabenbauer G, Lammert I, Jahnke K, Stueben G, Herrmann T, et al. Hyperfractionated accelerated chemoradiation with concurrent fluorouracil-mitomycin is more effective than dose-escalated hyperfractionated accelerated radiation therapy alone in locally advanced head and neck cancer: final results of the radiotherapy cooperative clinical trials group of the German Cancer Society 95-06 Prospective Randomized Trial. J Clin Oncol. 2005;23(6):1125-35.

4. Sartor $\mathrm{Cl}$. Biological modifiers as potential radiosensitizers: targeting the epidermal growth factor receptor family. Semin Oncol. 2000;27(6 Suppl 11):15-20. discussion 92-100.

5. Ang KK, Berkey BA, Tu X, Zhang HZ, Katz R, Hammond EH, Fu KK, Milas L. Impact of epidermal growth factor receptor expression on survival and pattern of relapse in patients with advanced head and neck carcinoma. Cancer Res. 2002;62(24):7350-6.

6. Huang SM, Bock JM, Harari PM. Epidermal growth factor receptor blockade with C225 modulates proliferation, apoptosis, and radiosensitivity in squamous cell carcinomas of the head and neck. Cancer Res. 1999;59(8):1935-40.

7. Milas L, Fan Z, Andratschke NH, Ang KK. Epidermal growth factor receptor and tumor response to radiation: in vivo preclinical studies. Int J Radiat Oncol Biol Phys. 2004:58(3):966-71.

8. Bonner JA, Harari PM, Giralt J, Azarnia N, Shin DM, Cohen RB, Jones CU, Sur R, Raben D, Jassem J, et al. Radiotherapy plus cetuximab for squamous-cell carcinoma of the head and neck. N Engl J Med. 2006;354(6):567-78.

9. Ang KK, Zhang Q, Rosenthal DI, Nguyen-Tan PF, Sherman EJ, Weber RS, Galvin JM, Bonner JA, Harris J, El-Naggar AK, et al. Randomized phase III trial of concurrent accelerated radiation plus cisplatin with or without cetuximab for stage III to IV head and neck carcinoma: RTOG 0522. Clin Oncol. 2014;32(27):2940-50.

10. Brandacher $G$, Perathoner A, Ladurner R, Schneeberger S, Obrist P, Winkler C, Werner ER, Werner-Felmayer G, Weiss HG, Gobel G, et al. Prognostic value of indoleamine 2,3-dioxygenase expression in colorectal cancer: effect on tumor-infiltrating T cells. Clin Cancer Res. 2006;12(4):1144-51.

11. Spranger S, Spaapen RM, Zha Y, Williams J, Meng Y, Ha TT, Gajewski TF. Up-regulation of PD-L1, IDO, and T(regs) in the melanoma tumor microenvironment is driven by CD8(+) T cells. Sci Transl Med. 2013:5(200):200ra116.

12. Ferris RL. Immunology and Immunotherapy of Head and Neck Cancer. J Clin Oncol. 2015;33(29):3293-304.

13. Grandis JR, Falkner DM, Melhem MF, Gooding WE, Drenning SD, Morel PA. Human leukocyte antigen class I allelic and haplotype loss in squamous cell carcinoma of the head and neck: clinical and immunogenetic consequences. Clin Cancer Res. 2000;6(7):2794-802.

14. Meissner $M$, Whiteside $T L$, van Kuik-Romein $P$, Valesky EM, van den Elsen $P J$, Kaufmann R, Seliger B. Loss of interferon-gamma inducibility of the MHC class II antigen processing pathway in head and neck cancer: evidence for post-transcriptional as well as epigenetic regulation. $\mathrm{Br} J$ Dermatol. 2008;158(5):930-40

15. Ferris RL, Whiteside TL, Ferrone S. Immune escape associated with functional defects in antigen-processing machinery in head and neck cancer. Clin Cancer Res. 2006;12(13):3890-5.

16. Jebreel A, Mistry D, Loke D, Dunn G, Hough V, Oliver K, Stafford N, Greenman J. Investigation of interleukin 10, 12 and 18 levels in patients with head and neck cancer. J Laryngol Otol. 2007;121(3):246-52.

17. Gastman BR, Johnson DE, Whiteside TL, Rabinowich H. Tumor-induced apoptosis of T lymphocytes: elucidation of intracellular apoptotic events. Blood. 2000;95(6):2015-23.

18. Jie HB, Gildener-Leapman N, Li J, Srivastava RM, Gibson SP, Whiteside TL, Ferris RL. Intratumoral regulatory T cells upregulate immunosuppressive molecules in head and neck cancer patients. Br J Cancer. 2013;109(10):2629-35.

19. Zandberg DP, Strome SE. The role of the PD-L1:PD-1 pathway in squamous cell carcinoma of the head and neck. Oral Oncol. 2014;50(7):627-32.

20. Yu GT, Bu LL, Huang CF, Zhang WF, Chen WJ, Gutkind JS, Kulkarni AB, Sun ZJ. PD-1 blockade attenuates immunosuppressive myeloid cells due to inhibition of CD47/SIRPalpha axis in HPV negative head and neck squamous cell carcinoma. Oncotarget. 2015;6(39):42067-80. 
21. Grubbe EH. The origin and birth of $x$-ray therapy. Urol Cutaneous Rev. 1947;51(7):375-9.

22. Burnette BC, Liang H, Lee $Y$, Chlewicki L, Khodarev NN, Weichselbaum RR, Fu YX, Auh SL. The efficacy of radiotherapy relies upon induction of type i interferon-dependent innate and adaptive immunity. Cancer Res. 2011;71(7):2488-96.

23. Lee $Y$, Auh SL, Wang Y, Burnette B, Wang Y, Meng Y, Beckett M, Sharma R, Chin $R$, Tu T, et al. Therapeutic effects of ablative radiation on local tumor require CD8+ $T$ cells: changing strategies for cancer treatment. Blood. 2009:114(3):589-95.

24. Gelbard A, Garnett CT, Abrams SI, Patel V, Gutkind JS, Palena C, Tsang KY, Schlom J, Hodge JW. Combination chemotherapy and radiation of human squamous cell carcinoma of the head and neck augments CTL-mediated Iysis. Clin Cancer Res. 2006;12(6):1897-905.

25. Stone $H B$, Peters $\sqcup$, Milas $L$. Effect of host immune capability on radiocurability and subsequent transplantability of a murine fibrosarcoma. J Natl Cancer Inst. 1979;63(5):1229-35.

26. Lugade AA, Moran JP, Gerber SA, Rose RC, Frelinger JG, Lord EM. Local radiation therapy of B16 melanoma tumors increases the generation of tumor antigen-specific effector cells that traffic to the tumor. J Immunol. 2005;174(12):7516-23.

27. Deng L, Liang H, Burnette B, Beckett M, Darga T, Weichselbaum RR, Fu YX. Irradiation and anti-PD-L1 treatment synergistically promote antitumor immunity in mice. J Clin Invest. 2014;124(2):687-95.

28. Galluzzi L, Maiuri MC, Vitale I, Zischka H, Castedo M, Zitvogel L, Kroemer G. Cell death modalities: classification and pathophysiological implications. Cell Death Differ. 2007;14(7):1237-43.

29. Golden EB, Apetoh L. Radiotherapy and immunogenic cell death. Semin Radiat Oncol. 2015;25(1):11-7.

30. Obeid M, Panaretakis T, Joza N, Tufi R, Tesniere A, van Endert P, Zitvogel L, Kroemer G. Calreticulin exposure is required for the immunogenicity of gamma-irradiation and UVC light-induced apoptosis. Cell Death Differ. 2007;14(10):1848-50

31. Yamazaki T, Hannani D, Poirier-Colame V, Ladoire S, Locher C, Sistigu A, Prada N, Adjemian S, Catani JP, Freudenberg M, et al. Defective immunogenic cell death of HMGB1-deficient tumors: compensatory therapy with TLR4 agonists. Cell Death Differ. 2014;21 (1):69-78.

32. Kroemer G, Galluzzi L, Kepp O, Zitvogel L. Immunogenic cell death in cancer therapy. Annu Rev Immunol. 2013;31:51-72.

33. Garnett CT, Palena C, Chakraborty M, Tsang KY, Schlom J, Hodge JW. Sublethal irradiation of human tumor cells modulates phenotype resulting in enhanced killing by cytotoxic T lymphocytes. Cancer Res. 2004;64(21):7985-94.

34. Lugade AA, Sorensen EW, Gerber SA, Moran JP, Frelinger JG, Lord EM. Radiation-induced IFN-gamma production within the tumor microenvironment influences antitumor immunity. J Immunol. 2008;180(5):3132-9.

35. Mole RH. Whole body irradiation; radiobiology or medicine? Br J Radiol. 1953;26(305):234-41.

36. Reynders K, Illidge T, Siva S, Chang JY, De Ruysscher D. The abscopal effect of local radiotherapy: using immunotherapy to make a rare event clinically relevant. Cancer Treat Rev. 2015;41(6):503-10.

37. Michot JM, Bigenwald C, Champiat S, Collins M, Carbonnel F, Postel-Vinay S, Berdelou A, Varga A, Bahleda R, Hollebecque A, et al. Immune-related adverse events with immune checkpoint blockade: a comprehensive review. Eur J Cancer. 2016;54:139-48.

38. Larkin J, Chiarion-Sileni V, Gonzalez R, Grob JJ, Cowey CL, Lao CD, Schadendorf D, Dummer R, Smylie M, Rutkowski P, et al. Combined Nivolumab and Ipilimumab or Monotherapy in Untreated Melanoma. N Engl J Med. 2015;373(1):23-34.

39. Seiwert TY, Burtness B, Mehra R, Weiss J, Berger R, Eder JP, Heath $K$, McClanahan T, Lunceford J, Gause C, et al. Safety and clinical activity of pembrolizumab for treatment of recurrent or metastatic squamous cell carcinoma of the head and neck (KEYNOTE-012): an open-label, multicentre, phase 1b trial. Lancet Oncol. 2016;17:956-65.

40. Bauml J, Seiwert TY, Pfister DG, Worden FP, Liu SV, Gillbert J, Saba NF, Weiss J, Wirth LJ, Sukari A, et al. Preliminary results from KEYNOTE-055: Pembrolizumab after platinum and cetuximab failure in head and neck squamous cell carcinoma (HNSCC). J Clin Oncol. 2016;34(suppl):abstr 6011.

41. Gillison ML, Blumenschein G, Fayette J, Guigay J, Colevas AD, Licitra L, Harrington K, Kasper S, Vokes E, Even C et al. Nivolumab (nivo) vs investigator's choice (IC) for recurrent or metastatic (R/M) head and neck squamous cell carcinoma (HNSCC): CheckMate-141 [abstract]. In: Proceedings of the 107th Annual Meeting of the American Association for Cancer Research. New Orleans: AACR; 2016.

42. Ferris RL, Blumenschein G, Fayette J, Guigay J, Colevas AD, Licitra L, Harrington K, Kasper S, Vokes E, Even C, et al. Further evaluations of nivolumab (nivo) versus investigator's choice (IC) chemotherapy for recurrent or metastatic (R/M) squamous cell carcinoma of the head and neck (SCCHN): CheckMate 141. J Clin Oncol. 2016;34(suppl):abstr 6009.

43. Herbst RS, Gordon MS, Fine GD, Sosman JA, Soria J-C, Hamid O, Powderly $J D$, Burris HA, Mokatrin A, Kowanetz M, et al. A study of MPDL3280A, an engineered PD-L1 antibody in patients with locally advanced or metastatic tumors. J Clin Oncol. 2013;31(Suppl):abstr 3000.

44. Segal NH, Antonia SJ, Brahmer JR, Maio M, Blake-Haskins A, Li X, Vasselli J, Ibrahim RA, Lutzky J, Khleif S. Preliminary data from a multi-arm expansion study of MEDI4736, an anti-PD-L1 antibody. J Clin Oncol. 2014;32(Suppl):abstr 3002.

45. Tokito T, Azuma K, Kawahara A, Ishii H, Yamada K, Matsuo N, Kinoshita T, Mizukami N, Ono H, Kage M, et al. Predictive relevance of PD-L1 expression combined with CD8+ TIL density in stage III non-small cell lung cancer patients receiving concurrent chemoradiotherapy. Eur J Cancer. 2016;55:7-14.

46. Chen MF, Chen PT, Chen WC, Lu MS, Lin PY, Lee KD. The role of PD-L1 in the radiation response and prognosis for esophageal squamous cell carcinoma related to IL-6 and T-cell immunosuppression. Oncotarget. 2016;7(7):7913-24.

47. Dovedi SJ, Adlard AL, Lipowska-Bhalla G, McKenna C, Jones S, Cheadle EJ, Stratford IJ, Poon E, Morrow M, Stewart R, et al. Acquired resistance to fractionated radiotherapy can be overcome by concurrent PD-L1 blockade. Cancer Res. 2014;74(19):5458-68.

48. Twyman-Saint Victor C, Rech AJ, Maity A, Rengan R, Pauken KE, Stelekati E, Benci JL, Xu B, Dada H, Odorizzi PM, et al. Radiation and dual checkpoint blockade activate non-redundant immune mechanisms in cancer. Nature. 2015;520(7547):373-7.

49. Chandra RA, Wilhite TJ, Balboni TA, Alexander BM, Spektor A, Ott PA, Ng AK, Hodi FS, Schoenfeld JD. A systematic evaluation of abscopal responses following radiotherapy in patients with metastatic melanoma treated with ipilimumab. Oncoimmunology. 2015;4(11):e1046028.

50. Distel LV, Fickenscher R, Dietel K, Hung A, Iro H, Zenk J, Nkenke E, Buttner M, Niedobitek G, Grabenbauer GG. Tumour infiltrating lymphocytes in squamous cell carcinoma of the oro- and hypopharynx: prognostic impact may depend on type of treatment and stage of disease. Oral Oncol. 2009;45(10):167-74.

51. Balermpas P, Michel Y, Wagenblast J, Seitz O, Weiss C, Rodel F, Rodel C, Fokas E. Tumour-infiltrating lymphocytes predict response to definitive chemoradiotherapy in head and neck cancer. Br J Cancer. 2014;110(2):501-9.

52. Oguejiofor K, Hall J, Slater C, Betts G, Hall G, Slevin N, Dovedi S, Stern PL, West CM. Stromal infiltration of CD8 T cells is associated with improved clinical outcome in HPV-positive oropharyngeal squamous carcinoma. Br J Cancer. 2015;113(6):886-93.

53. Balermpas P, Rodel F, Rodel C, Krause M, Linge A, Lohaus F, Baumann M, Tinhofer I, Budach V, Gkika E, et al. CD8+ tumour-infiltrating lymphocytes in relation to HPV status and clinical outcome in patients with head and neck cancer after postoperative chemoradiotherapy: A multicentre study of the German cancer consortium radiation oncology group (DKTK-ROG). Int J Cancer. 2016;138(1):171-81.

54. Patel SP, Kurzrock R. PD-L1 Expression as a Predictive Biomarker in Cancer Immunotherapy. Mol Cancer Ther. 2015;14(4):847-56.

55. Seiwert TY, Haddad RI, Gupta S, Mehra R, Tahara M, Berger R, Lee S-H, Burtness B, Le DT, Heath $\mathrm{K}$, et al. Antitumor activity and safety of pembrolizumab in patients (pts) with advanced squamous cell carcinoma of the head and neck (SCCHN): Preliminary results from KEYNOTE-012 expansion cohort. J Clin Oncol. 2015;33(Suppl):abstr LBA6008.

56. Ribas R, Robert C, Hodi FS, Wolchok JD, Joshua AM, Hwu WJ, Weber JS, Zarour HM, Kefford R, Loboda A, et al. Association of response to programmed death receptor 1 (PD-1) blockade with pembrolizumab (MK-3475) with an interferon-inflammatory immune gene signature. J Clin Oncol. 2015;33(Suppl):abstr 3001.

57. Shankaran V, Muro K, Bang Y-J, Geva R, Catenacci DVT, Gupta S, Eder JP, Berger R, Loboda A, Albright A, et al. Correlation of gene expression signatures and clinical outcomes in patients with advanced gastric cancer treated with pembrolizumab (MK-3475). J Clin Oncol. 2015;33(suppl):abstr 3026.

58. Rosenberg JE, Hoffman-Censits J, Powles T, van der Heijden MS, Balar AV, Necchi A, Dawson N, O'Donnell PH, Balmanoukian A, Loriot Y, et al. 
Atezolizumab in patients with locally advanced and metastatic urothelial carcinoma who have progressed following treatment with platinum-based chemotherapy: a single-arm, multicentre, phase 2 trial. Lancet. 2016:387(10031):1909-20.

59. Le DT, Uram JN, Wang H, Bartlett BR, Kemberling H, Eyring AD, Skora AD, Luber BS, Azad NS, Laheru D, et al. PD-1 Blockade in Tumors with Mismatch-Repair Deficiency. N Engl J Med. 2015;372(26):2509-20.

60. Martins I, Kepp O, Schlemmer F, Adjemian S, Tailler M, Shen S, Michaud M, Menger L, Gdoura A, Tajeddine N, et al. Restoration of the immunogenicity of cisplatin-induced cancer cell death by endoplasmic reticulum stress. Oncogene. 2011;30(10):1147-58.

61. Hodge JW, Garnett CT, Farsaci B, Palena C, Tsang KY, Ferrone S, Gameiro SR. Chemotherapy-induced immunogenic modulation of tumor cells enhances killing by cytotoxic T lymphocytes and is distinct from immunogenic cell death. Int J Cancer. 2013;133(3):624-36.

62. Golden EB, Frances D, Pellicciotta I, Demaria S, Helen Barcellos-Hoff M, Formenti SC. Radiation fosters dose-dependent and chemotherapy-induced immunogenic cell death. Oncoimmunology. 2014;3:e28518.

63. Lesterhuis WJ, Punt CJ, Hato SV, Eleveld-Trancikova D, Jansen BJ, Nierkens S, Schreibelt G, de Boer A, Van Herpen CM, Kaanders JH, et al. Platinum-based drugs disrupt STAT6-mediated suppression of immune responses against cancer in humans and mice. J Clin Invest. 2011;121(8):3100-8.

64. Srivastava RM, Lee SC, Andrade Filho PA, Lord CA, Jie HB, Davidson HC, Lopez-Albaitero A, Gibson SP, Gooding WE, Ferrone S, et al. Cetuximabactivated natural killer and dendritic cells collaborate to trigger tumor antigen-specific T-cell immunity in head and neck cancer patients. Clin Cancer Res. 2013;19(7):1858-72.

65. Mendenhall WM, Amdur RJ, Palta JR. Intensity-modulated radiotherapy in the standard management of head and neck cancer: promises and pitfalls. J Clin Oncol. 2006;24(17):2618-23.

66. Seiwert TY, Salama JK, Vokes EE. The chemoradiation paradigm in head and neck cancer. Nat Clin Pract Oncol. 2007;4(3):156-71.

67. Hodi FS, O'Day SJ, McDermott DF, Weber RW, Sosman JA, Haanen JB, Gonzalez R, Robert C, Schadendorf D, Hassel JC, et al. Improved survival with ipilimumab in patients with metastatic melanoma. N Engl J Med. 2010;363(8):711-23.

68. Gangadhar TC, Vonderheide RH. Mitigating the toxic effects of anticancer immunotherapy. Nat Rev Clin Oncol. 2014;11(2):91-9.

\section{Submit your next manuscript to BioMed Central and we will help you at every step:}

- We accept pre-submission inquiries

- Our selector tool helps you to find the most relevant journal

- We provide round the clock customer support

- Convenient online submission

- Thorough peer review

- Inclusion in PubMed and all major indexing services

- Maximum visibility for your research

Submit your manuscript at www.biomedcentral.com/submit

) Biomed Central 